\title{
Avaliação do Equilíbrio Postural em Pacientes com DPOC com o BESTest antes e após um atendimento de Fisioterapia
}

\author{
Postural Balance Evaluation in Patients with COPD with BESTest before and after a \\ Physiotherapy Care
}
Evaluación del Equilibrio Postural en Pacientes con EPOC con BESTest antes y después de una atención de Fisioterapia

Vívian Michele Lopes Cruz ${ }^{1 *}$, Dayara Aparecida Nogueira ${ }^{1}$, Gislene Guimarães Garcia Tomazini ${ }^{1}$, Pâmela Camila Pereira ${ }^{1}$.

\section{RESUMO}

Objetivo: Avaliar o equilíbrio postural em pacientes com DPOC com a BESTest antes e após um atendimento de Fisioterapia. Métodos: Trata-se de um estudo de ensaio clínico, prospectivo, descritivo e experimental, realizado na Clínica Médica da Santa Casa de Misericórdia de Itajubá-MG. Os pacientes foram selecionados e submetidos a uma avaliação minuciosa, contendo dados sociodemográficos e avaliação do equilíbrio com - BESTest. Em seguida, foi aplicado um protocolo de exercícios para reabilitação do equilíbrio do paciente no mesmo atendimento. Resultado: Participaram do estudo 8 pacientes do sexo feminino e 1 do sexo masculino. Todos os pacientes apresentaram alteração do equilíbrio postural ao serem avaliados com o BESTest. Após a aplicação do protocolo, foi possível verificar a melhora do equilíbrio postural nos sistemas: Limites de estabilidade $(p=0,015)$, Transições posturais $(p=0,008)$, Orientação sensorial $(p=0,0002)$ e Estabilidade na Marcha $(p=0,016)$. Conclusão: $A$ utilização do BESTest permite uma avaliação mais detalhada dos sistemas em que o paciente apresenta maiores déficits, com isso a atuação da fisioterapia torna-se mais específica. A reabilitação fisioterapêutica é eficaz na melhora do equilíbrio postural, mesmo com seu efeito agudo em pacientes com DPOC.

Palavras-chave: Doença Pulmonar Obstrutiva Crônica, Equilíbrio Postural, Acidentes por Quedas.

\begin{abstract}
Objective: To evaluate the postural balance in patients with COPD with BESTest before and after a physicaltherapy care. Methods: This is a clinical, prospective, descriptive and experimental study conducted at the Santa Casa de Misericórdia Medical Clinic in Itajubá-MG. The patients were selected and submitted to a thorough evaluation, containing sociodemographic data and balance evaluation with BESTest. Then, an exercise protocol was applied to rehabilitate the patient's balance in the same care. Results: Eight female patients and one male patient participated in the study. All patients presented altered postural balance when evaluated with BESTest. After the application of the protocol, it was possible to verify the improvement of the postural balance in the systems: Stability limits $(p=0.015)$, Postural Transitions $(p=0.008)$, Sensory orientation $(p=0.0002)$ and Stability in gait $(p=0.016)$. Conclusion: The use of BESTest allows a more detailed evaluation of the systems in which the patient presents major deficits, with the result that the physiotherapy performance becomes more specific. The Physiotherapy rehabilitation is effective in improving postural balance, even with its acute effect in patients with COPD.
\end{abstract}

Keywords: Chronic Obstructive Pulmonar Disease, Postural Balance, Accidental Falls.

${ }^{1}$ Fisioterapeuta, Centro Universitário de Itajubá (FEPI), Itajubá-MG * E-mail: vivian cruz09@hotmail.com 


\section{RESUMEN}

Objetivo: Evaluar el balance postural en pacientes con EPOC con BESTest antes y después de una atención de Fisioterapia. Métodos: Se trata de un estudio de ensayo clínico, prospectivo, descriptivo y experimental, realizado en la Clínica Médica de la Santa Casa de Misericordia de Itajubá-MG. Los pacientes fueron seleccionados y sometidos a una evaluación minuciosa, conteniendo datos sociodemográficos y evaluación del balance con el BESTest. A continuación, se aplicó un protocolo de ejercicios para rehabilitación del balance del paciente en la misma atención. Resultado: Participaron del estudio 8 pacientes del sexo femenino y 1 del sexo masculino. Todos los pacientes presentaron alteración del balance postural al ser evaluados con el BESTest. Después de la aplicación del protocolo, fue posible verificar la mejora del balance postural en los sistemas: Límites de estabilidad $(p=0,015)$, Transiciones posturales $(p=0,008)$, Orientación sensorial $(p=0,0002)$ y Estabilidad en la Marcha $(p=0,016)$. Conclusión: La utilización de BESTest permite una evaluación más detallada de los sistemas en los que el paciente presenta mayores déficits, con lo que la actuación de la fisioterapia se vuelve más específica. La rehabilitación fisioterapéutica es eficaz en la mejora del balance postural, incluso con su efecto agudo en pacientes con EPOC.

Palavras-clave: Enfermedad Pulmonar Obstructiva Crónica, Balance Postural, Accidentes por Caídas.

\section{INTRODUÇÃO}

Segundo Nogueira LV et al. (2017), o indivíduo é considerado idoso em países em desenvolvimento essa definição ocorre a partir dos 60 anos. O Brasil apresenta crescente taxa da população idosa, no qual a proporção de idosos aumentou de 15,5 milhões em 2001, para 23,5 milhões em 2011 (VALÉRIO RBC e DUTRA FCMS, 2016).

O envelhecimento é caracterizado por um conjunto de alterações morfológicas, fisiológicas, bioquímicas, psicológicas, acarretando na perda progressiva da capacidade de adaptação ao meio ambiente afetando a capacidade do indivíduo em realizar tarefas cotidianas podendo levar a alta incidência de quedas que implica negativamente a qualidade de vida dos idosos (NOGUEIRA LV, et al., 2017; TAGUCHI CK, et al., 2016).

A queda é caracterizada pelo deslocamento do corpo não intencional sem que haja capacidade para correção em tempo hábil. A queda ocorre por circunstâncias multifatoriais, sendo resultante da interação de fatores intrínsecos como as modificações fisiológicas próprias do envelhecimento como a diminuição da acuidade visual e da audição, as alterações posturais, a diminuição de massa muscular e da força e o equilíbrio, que em conjunto afetam a marcha e, os fatores extrínsecos relacionados aos riscos ambientais como má iluminação, piso escorregadio e exposições perigosas em atividades do cotidiano (VIEIRA LS, et al., 2018; NOGUEIRA LV, et al., 2017).

Tais modificações são comuns em pacientes com Doença Pulmonar Obstrutiva Crônica (DPOC), que é uma doença crônica comum, caracterizada pela limitação do fluxo de ar causada pelo acometimento das pequenas vias aéreas e a destruição do parênquima pulmonar, consequentes da exposição exacerbada à partículas e gases nocivos. Embora a DPOC seja uma enfermidade que acomete os pulmões, suas consequências sistêmicas são significativas, pois a dispneia gerada pelas alterações no parênquima pulmonar leva à intolerância de realizar exercícios físicos e as atividades de vida diária e, consequentemente, os pacientes começam a apresentar sedentarismo crônico (GOLD, 2017; DIAS FD, et al., 2014).

Pacientes com DPOC apresentam a marcha afetada, devido ao cansaço em poucos minutos de caminhada. Devido a debilidade, os pacientes podem apresentar déficit de equilíbrio e alteração na deambulação, podendo ser um fator de risco para quedas. As quedas são caracterizadas com uma das maiores causas de morbidade para os idosos, pois podem gerar síndrome de fragilidade, perda da autonomia e alteração no estado psicossocial (FIEL JNA, et al., 2018). 
Segundo Porto EF et al. (2015) os fatores que contribuem para a disfunção do controle postural são: inatividade física, idade avançada, mobilidade limitada e uso de oxigênio auxiliar. Em consequência disso, a fraqueza muscular periférica, as doenças labirínticas, o déficit de desempenho funcional e as alterações de equilíbrio aumentam o risco de quedas nesses pacientes. As quedas estão associadas ao aumento da mortalidade, diminuição da independência e nível de atividade física, e piora da qualidade de vida. Devido a isso, é fundamental avaliar o equilíbrio desses indivíduos, pois ao detectar um déficit, as medidas serão tomadas precocemente, melhorando a qualidade de vida dos idosos e reduzindo o risco de quedas da população (LEME GLM, et al., 2017).

No decorrer dos anos, foram desenvolvidos diversos instrumentos para medir quantitativamente o equilíbrio na população idosa. Estes instrumentos de rastreio são utilizados para avaliar a capacidade de manter o equilíbrio e, posteriormente, para identificar o risco de queda em um futuro próximo. Com isso, o Balance Evaluation Systems Test (BESTest) foi desenvolvido em 2009 para identificar qual sistema de controle de equilíbrio está deficitário e auxilia a direcionar especificamente o tratamento, sendo um instrumento válido, confiável e valioso para identificar os déficits de equilíbrio e risco de queda em pacientes com DPOC (JÁCOME C, et al., 2016; CRIŞAN AF, et al., 2015; RODRIGUES LC et al., 2014; MAIA AC, et al., 2013).

Portanto, o objetivo do presente estudo foi avaliar o equilíbrio postural em pacientes com DPOC com a BESTest antes e após um atendimento de Fisioterapia.

\section{MÉTODOS}

Trata-se de um estudo de ensaio clínico, prospectivo, descritivo e experimental, realizado no ambulatório da Clínica Médica da Santa Casa de Misericórdia de Itajubá - MG, localizada na Avenida Cesário Alvim, 632, Centro de Itajubá, Minas Gerais. Aprovado pelo Comitê de Ética e Pesquisa do Centro Universitário de Itajubá - FEPI, sob o parecer de no $2.594 .376 / 2018$.

Foram incluídos nesse estudo 9 pacientes com diagnóstico de DPOC, idade entre 60 e 85 anos, ambos os sexos e com capacidade de deambular.

Após o consentimento do paciente ou do responsável conforme a resolução $n^{\circ} 466 / 12$ do conselho Nacional de Saúde, foi realizada uma avaliação minuciosa com a aplicação de um questionário sociodemográfico composto por dados pessoais e profissionais do paciente.

Em seguida, foi realizada a avaliação do equilíbrio com o instrumento Balance Evaluation Systems Test BESTest. A BESTest é uma escala de avaliação para mensurar o equilíbrio, composta por 36 itens agrupados em seis sistemas: restrições biomecânicas, limites de estabilidade/verticalidade, ajustes posturais antecipatórios, respostas posturais, orientação sensorial e estabilidade na marcha. Cada tarefa é avaliada em número ordinais que vai de 0 (pior equilíbrio) à 3 (melhor equilíbrio). A pontuação total do BESTest é de 108 pontos, a pontuação é feita por percentual (0-100\%), quanto maior a pontuação, melhor é o desempenho do equilíbrio (DE ALMEIDA SIL, et al., 2017).

Após identificar os déficits de equilíbrio, foi aplicado um protocolo de exercícios (Quadro 1) para reabilitação do equilíbrio do paciente no mesmo atendimento. Ao final do protocolo, o equilíbrio foi reavaliado. Cada atendimento teve a duração de 1 hora e cada paciente foi atendido em dias diferentes.

Os dados foram dispostos em tabelas e gráficos confeccionadas no programa Excel (2016). Em seguida, foi realizada a análise estatística no pacote estatístico GraphPad Prism versão 7.0. A normalidade dos dados foi verificada por meio do teste de Shapiro-Wilk. Para averiguar a diferença entre o equilíbrio nos sistemas de Restrições Biomecânicas, Limitações de Estabilidade, Respostas Posturais e Estabilidade na Marcha antes e após o protocolo utilizou-se o teste Wilcoxon. Para verificar a diferença das Transições e Orientação Sensorial antes e após o protocolo utilizou-se o teste T. A significância adotada para este estudo foi de $(p<0,05)$. 
Quadro 1 - Protocolo de Reabilitação do Equilíbrio.

\begin{tabular}{|c|}
\hline Exercícios Semi Estáticos - Solo Estável \\
\hline $\begin{array}{l}\text { 1- Em ortostatismo com a base estreita, mantendo a postura por } 30 \text { segundos. } \\
\text { 2- Em ortostatismo com os olhos fechados, mantendo por } 20 \text { segundos. } \\
\text { 3- Em apoio unipodal, por } 30 \text { segundos. }\end{array}$ \\
\hline Exercícios Semi Estáticos - Solo Instável \\
\hline $\begin{array}{l}\text { 1- Em ortostatismo sobre a espuma, mantendo por } 20 \text { segundos. } \\
\text { 2- Em ortostatismo sobre a espuma com olhos fechados, durante } 20 \text { segundos. } \\
\text { 3- Em ortostatismo sobre a espuma com a base reduzida, durante por } 20 \text { segundos. } \\
\text { 4- Em apoio unipodal sobre a espuma, mantendo por } 20 \text { segundos. }\end{array}$ \\
\hline Exercícios de Transição \\
\hline $\begin{array}{l}\text { 1- Sedestação para ortostatismo em uma cadeira com braços, } 5 \text { repetições. } \\
\text { 2- Sedestação para ortostatismo em uma cadeira sem braços, } 5 \text { repetições. }\end{array}$ \\
\hline Exercícios de Marcha \\
\hline $\begin{array}{l}\text { 1- Marcha lateral com faixa elástica, } 10 \text { repetições. } \\
\text { 2- Marcha com estímulos visuais para oscilação da cabeça com flexão e extensão, rotações e } \\
\text { dupla tarefa (marcha com estímulos verbais, com nome de frutas, nomes pessoais e de animais). }\end{array}$ \\
\hline Exercícios Funcionais \\
\hline $\begin{array}{l}\text { 1- Agachamento com apoio de braço, } 10 \text { repetições. } \\
\text { 2- Subir e descer um degrau, } 10 \text { repetições. } \\
\text { 3- Sentar-se sobre a bola suíça, manter a postura por } 60 \text { segundos. }\end{array}$ \\
\hline
\end{tabular}

Fonte: Dados da pesquisa, 2018

\section{RESULTADOS}

A caracterização da amostra estudada foi realizada com o questionário sócio demográfico, no qual contém informações especificas sobre os pacientes, representada na Tabela 1.

Em relação ao tabagismo, a maioria dos pacientes relatou ser ex-tabagista. Uma distribuição mais detalhada pode ser observada no Gráfico 1.

Todos os pacientes avaliados apresentaram alteração do equilíbrio ao serem avaliados com o BESTest como demonstrado no Gráfico 2. 
Tabela 1- Dados sociodemográficos dos pacientes.

\begin{tabular}{lc}
\hline Dados & $\mathbf{n ~ ( \% )}$ \\
\hline Sexo & $8(89)$ \\
Feminino & $1(11)$ \\
Masculino & \\
\hline Faixa Etária & $4(44)$ \\
60 a 69 anos & $3(33)$ \\
70 a 79 anos & $2(22)$ \\
80 a 85 anos & \\
\hline Estado Civil & 0 \\
Solteiro (a) & $4(44)$ \\
Casado (a) & $5(56)$ \\
Viúvo (a) & $9(100)$ \\
\hline Escolaridade & \\
Ensino Fundamental & $5(56)$ \\
\hline Aposentado & $4(44)$ \\
Sim & $5(56)$ \\
Não & $4(44)$ \\
\hline Exposição Laboral & \\
Sim & Não
\end{tabular}

Fonte: Dados da pesquisa, 2018

Gráfico 1- Distribuição em relação ao tabagismo

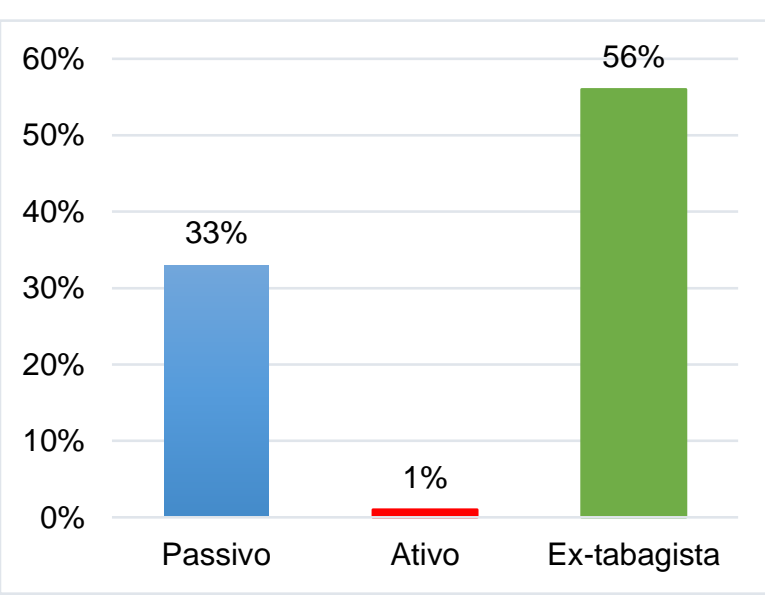

Fonte: Dados da pesquisa, 2018
Gráfico 2 - Avaliação do Equilíbrio com a BESTest

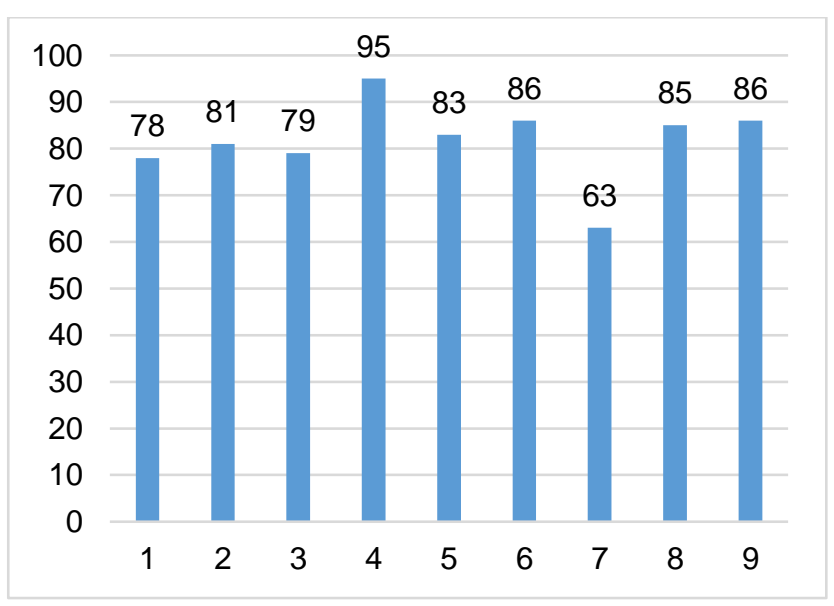

Fonte: Dados da pesquisa, 2018 
Após a identificação das alterações do equilíbrio, os déficits foram estratificados separadamente nos sistemas da BESTest e foram analisados após a reabilitação do equilíbrio (Tabela 2).

Tabela 2 - Sistemas do BESTest

\begin{tabular}{cc}
\hline BESTest & Valor $\mathbf{p}$ \\
\hline Restrições Biomecânicas & $\mathrm{p}=0,125$ \\
Estabilidade & $\mathrm{p}=0,015^{\star}$ \\
Transições Posturais & $\mathrm{p}=0,008^{\star}$ \\
Respostas Posturais & $\mathrm{p}=0,313$ \\
Orientação sensorial & $\mathrm{p}=0,0002^{\star}$ \\
Estabilidade na Marcha & $\mathrm{p}=0,016^{*}$
\end{tabular}

Fonte: Dados da pesquisa, 2018

A eficácia do protocolo de exercícios na reabilitação do equilíbrio foi comprovada somente em um estudo, que observou melhora significativa $(p<0,01)$ no equilíbrio, após aplicar o protocolo de 18 sessões com 21 pacientes diagnosticados com DPOC, avaliados pelo BESTest (BEAUCHAMP MK, et al., 2013), tais dados corroboram com os resultados obtidos nessa pesquisa. Entretanto, cabe ressaltar que apenas o presente estudo demonstrou a melhora significativa do equilíbrio postural em pacientes com DPOC após somente uma sessão de fisioterapia.

\section{DISCUSSÃO}

Pacientes com DPOC no decorrer da doença, apresentam debilidade e, com isso, podem apresentar déficit de equilíbrio e alteração na deambulação, sendo consequentemente, um fator de risco para quedas (FIEL JNA, et al., 2018). No presente estudo foi verificada a alteração do equilíbrio e consequente risco de quedas em pacientes com DPOC em todos os pacientes analisados. Tais dados corroboram com o estudo de CRIŞAN AF, et al. (2015), no qual foram avaliados 29 pacientes com DPOC estável e 17 pacientes com exacerbação da doença e verificaram que a presença da DPOC está associada ao déficit de equilíbrio e o aumento do risco de quedas.

Em relação aos déficits, após a aplicação do protocolo, os pacientes apresentaram melhora significativa nos sistemas: limites de estabilidade, transições posturais, orientação sensorial e estabilidade da marcha. $O$ estudo de Beauchamp MK et al. (2013) obteve dados semelhantes ao reabilitar o equilíbrio de 39 pacientes com DPOC, encontrou melhorias biomecânica, transições, reativos, controle e marcha baseados em observações prévias de seu comprometimento na DPOC.

No presente estudo após a reabilitação do equilíbrio não houve melhora significativa no sistema de restrições biomecânicas, que está relacionado à redução da força muscular de membros inferiores. Segundo Dias FD et al. (2014) o desuso da musculatura, principalmente a periférica, devido a dispneia intensa apresentada pelos pacientes com DPOC durante as atividades físicas, leva a uma atrofia muscular e quanto mais grave for este paciente, menor será a capacidade funcional em decorrência do menor recrutamento muscular durante suas atividades diárias.

O equilíbrio postural em pacientes com DPOC foi avaliado também por Oliveira CC et al. (2017), Voica AS et al. (2016), Castro LAC et al. (2016) e Crişan AF et al. (2015), no qual foram encontrados déficits no equilíbrio 
semelhantes à presente pesquisa, porém a ferramenta de avaliação diferiu entre os estudos, pois os estudos utilizaram a Berg Balance Scale e o Timed Up and Go Test.

No presente estudo houve predominância de pacientes do sexo feminino (89\%). Dados que são semelhantes ao estudo de Barbosa ATF et al. (2017), no qual houve predomínio do sexo feminino com 65,9\% da amostra. Tal fato é explicado por Rabahi MF (2013), no sexo feminino, a DPOC apresenta-se mais sintomática e mais grave, demostrando um aumento proporcional de mortalidade entre as mulheres, refletido pela tendência mundial do aumento proporcional do tabagismo entre essas, no qual a prevalência de DPOC em mulheres ultrapassou a dos homens (6,7\% vs. 5,2\%).

Em relação ao tabagismo, 89\% dos pacientes tiveram exposição ao tabaco no presente estudo, sendo que $56 \%$ da amostra era ex-tabagista. No estudo de Barbosa ATF et al. (2017), ao analisar 179 idosos com DPOC, encontrou uma relação entre o tabagismo sedo atual ou prévio com a presença de DPOC nos idosos, sem ter evidenciado associação com a carga tabágica.

Uma das limitações encontradas no presente estudo foi a dificuldade em encontrar idosos do sexo masculino hospitalizados com DPOC e, assim, incapacitando de fazer comparações com números iguais entre homens e mulheres. O ponto forte deste estudo foi a utilização de ferramentas de fácil aplicação e fidedignas para utilizar na prática clínica.

\section{CONCLUSÃO}

A utilização do BESTest permite uma avaliação mais detalhada dos sistemas em que o paciente apresenta maiores déficits, com isso a atuação da fisioterapia torna-se mais específica. A reabilitação fisioterapêutica mostrou-se efetiva na melhora do equilíbrio postural em pacientes com DPOC, apresentando resultados positivos no escore de equilíbrio postural, mesmo com seu efeito agudo em pacientes com DPOC.

\section{REFERÊNCIAS}

1- BARBOSA ATF, et al. Fatores associados à Doença Pulmonar Obstrutiva Crônica em idosos. Ciência \& Saúde Coletiva, 2017; 22(1), 63-73.

2- BEAUCHAMP MK, et al. Randomized Controlled Trial of Balance Training During Pulmonary Rehabilitation for Individuals With COPD. CHEST, Dec 2013; 144(6), 1803-1810.

3- CASTRO LAC, et al. Static and Functional Balance in Individuals With COPD: Comparison With Healthy Controls and Differences According to Sex and Disease Severity. Respiratory Care, November 2016; 61(11), $1488-1496$.

4- CRIŞAN AF, et al. Balance Impairment in Patients with COPD, Plos One, 2015; March 13, 10(3), 1-11.

5- DE ALMEIDA SIL, et al. Normative values of the Balance Evaluation System Test (BESTest), Mini-BESTest, Brief-BESTest, Timed Up and Go Test and Usual Gait Speed in healthy older Portuguese People. Rev Port Med Geral Fam, 2017; 33, 106-116.

6- DIAS FD, et al. Avaliação da composição corporal, capacidade funcional e função pulmonar em pacientes com Doença Pulmonar Obstrutiva Crônica. Fisioter Pesq, 2014; 21(1), 10-15.

7- FIEL JNA, et al. Avaliação do risco de quedas e sarcopenia em idosos com doença pulmonar obstrutiva crônica atendidos em um hospital universitário de Belém, Estado do Pará, Brasil. Rev Pan-Amaz Saude, 2016, 7(4), 4145.

8- GLOBAL INITIATIVE FOR CHRONIC OBSTRUCTIVE LUNG DISEASE (GOLD). Global Strategy for the Diagnosis, Management and Prevention of COPD, 2017. Disponível em: http://goldcopd.org.

9- JÁCOME C, et al. Validity, reliability, and ability to identify fall status of the Berg Balance Scale, BESTest, MiniBESTest, and Brief-BESTest in patients with COPD. Phys Ther., 2016, 96, 1807-1815.

10- LEME GLM, et al. Melhora do equilíbrio postural em mulheres idosas com o uso de informação sensorial adicional. Fisioter Pesqui, 2017; 24(1), 68-73.

11- MAIA AC, et al. Cross-cultural adaptation and analysis of the psychometric properties of the Balance Evaluation Systems Test and MiniBESTest in the elderly and individuals with Parkinson's disease: application of the Rasch model. Braz J Phys Ther, May-June, 2013; 17(3), 195-217.

12- NOGUEIRA LV, et al. Risco de quedas e capacidade funcional em idosos. Rev Soc Bras Clin Med, 2017 abrjun;15(2):90-3. 
13- OLIVEIRA CC, et al. Balance and Falls in Acute Exacerbation of Chronic Obstructive Pulmonary Disease: A Prospective Study. COPD: Journal of Chronic Obstructive Pulmonary Disease, 2017; 1-8.

14- PORTO EF, et al. Postural control in chronic obstructive pulmonary disease: a systematic review. International Journal of COPD, 2015, 10, 1233-1239.

15- RABAHI MF. Epidemiologia da DPOC: Enfrentando Desafios. Pulmão RJ, 2013; 22(2), 4-8.

16- RODRIGUES LC, et al. Reliability of the Balance Evaluation Systems Test (BESTest) and BESTest sections for adults with hemiparesis. Braz J Phys Ther, 2014; May-June, 18(3), 276-281.

17- TAGUCHI CK, et al. Quality of Life and Gait in Elderly Group. International Archives of Otorhinolaryngology, 2016; 20(3), 235-240.

18- VALÉRIO RBC, DUTRA FCMS. Envelhecimento Funcional e Capacidade para o Trabalho entre Trabalhadores Atendidos pela Atenção Básica. Ciencia \& Trabajo, 2016; Septiembre / Diciembre, 18(57), 190-195.

19- VIEIRA LS, et al. Quedas em idosos no Sul do Brasil: prevalência e determinantes. Rev Saude Publica, 2018; 52(22), 1-13.

20- VOICA AS, et al. Chronic obstructive pulmonary disease phenotypes and balance impairment. International Journal of COPD, 2016; 11, 919-925. 